Check for updates

Cite this: Nanoscale Adv., 2019, 1, 1215

\title{
Pulsed-grown graphene for flexible transparent conductors $\uparrow$
}

\author{
Pramoda K. Nayak iD *
}

In the race to find novel transparent conductors for next-generation optoelectronic devices, graphene is supposed to be one of the leading candidates, as it has the potential to satisfy all future requirements. However, the use of graphene as a truly transparent conductor remains a great challenge because its lowest sheet resistance demonstrated so far exceeds that of the commercially available indium tin oxide. The possible cause of low conductivity lies in its intrinsic growth process, which requires further exploration. In this work, I have approached this problem by controlling graphene nucleation during the chemical vapor deposition process as well as by adopting three distinct procedures, including bis(trifluoromethanesulfonyl)amide doping, post annealing, and flattening of graphene films. Additionally, van der Waals stacked graphene layers have been prepared to reduce the sheet resistance effectively. I have demonstrated an efficient and flexible transparent conductor with the extremely low sheet resistance of $40 \Omega \mathrm{sq}^{-1}$, high transparency $\left(T_{\mathrm{r}} \sim 90 \%\right)$, and high mechanical flexibility, making it suitable for electrode materials in future optoelectronic devices.

Received 30th August 2018

Accepted 1st January 2019

DOI: $10.1039 / c 8 n a 00181 b$

rsc.li/nanoscale-advances
Significant efforts have been made so far to reduce the $R_{\mathrm{s}}$ of graphene film by chemical doping, ${ }^{17-19}$ substitutional doping, ${ }^{20-22}$ or stacking of graphene films ${ }^{7,23,24}$ in parallel while maintaining a high $T_{\mathrm{r}}$ to improve its potential to be harnessed in optoelectronic applications. Although chemical doping has been shown to effectively reduce the $R_{\mathrm{S}}$ of graphene down to 150 $\Omega \mathrm{sq}^{-1}$ at $T_{\mathrm{r}}=87 \%,{ }^{12} 125 \Omega \mathrm{sq}^{-1}$ at $T_{\mathrm{r}}=97.4 \%,{ }^{13}$ and $80 \Omega \mathrm{sq}^{-1}$ at $T_{\mathrm{r}}=80 \%$ for eight-layer stacked graphene, ${ }^{7}$ the adsorption of moisture, other atoms, and molecules onto graphene after chemical treatment leads to the increase of $R_{\mathrm{S}}$ within few days. ${ }^{25,26}$ Similarly, substitutional doping induces disorder in the graphene, thereby reducing its mobility as a result of scattering of Dirac electrons. ${ }^{27,28}$ Additionally, most doping processes decrease the optical transparency of graphene film by changing its band structure or by forming optically reflective nanoparticles at the surface, ${ }^{29,30}$ making it unsuitable for optoelectronic applications. Recently, a new growth technique has been introduced by Han et al. ${ }^{31}$ which involves cyclic injection of carbon precursor $\left(\mathrm{CH}_{4}\right)$ during chemical vapor deposition (CVD) to suppress the multilayer patches generally formed using continuous $\mathrm{CH}_{4}$ flow, thereby improving both the optical and electrical performance of graphene. Similarly, other approaches, such as trifluoromethanesulfonimide (TFSA) doping, ${ }^{19,32-34}$ post annealing, ${ }^{35,36}$ and flattening of graphene films ${ }^{37}$ have been carried out to reduce the $R_{\mathrm{s}}$ of graphene. However, no systematic study exists so far that combines all the aforementioned approaches, which is highly desired to achieve superior graphene film suitable for the transparent conducting electrode.

In the present work, I have prepared a high-quality graphene film using pulsed-grown technique and investigated its optical

Department of Physics, Indian Institute of Technology Madras, Chennai 600036, India

(ESI) available: Experimental reduce sheet resistance, graphene transfer process, sheet resistan measurement, flexibility test and reduction of sheet resistance in continuous
} 
and electrical performance using the combined effects of TFSA doping, stacking, post annealing and flattening. The prepared graphene film shows $R_{\mathrm{s}}$ of a few hundred $\Omega \mathrm{sq}^{-1}$, on/off ratio of $\sim 13$, along with a high $I_{2 \mathrm{D}} / I_{\mathrm{G}}$ ratio (>1.5). TFSA doped four layer stacked graphene films show a low $R_{\mathrm{S}}$ of $40 \Omega \mathrm{sq}^{-1}$ and transmittance of $90 \%$, which are almost comparable to those of ITO. The combination of TFSA doping, post annealing and flattening of graphene film is found to reduce the $R_{\mathrm{S}}$ by $80 \%$, the highest value reported so far. Finally, the mechanical flexibility of this highly conducting and transparent film has been demonstrated, making it suitable for application as a flexible transparent conducting electrode.

\section{Results and discussion}

Growth of monolayer graphene on Cu substrate using CVD has been a well-established technique since $2009,{ }^{38-40}$ where the nucleation and growth of graphene usually occurs by exposure of the $\mathrm{Cu}$ surface to a hydrocarbon gas under atmospheric pressure, low pressure or ultra-high vacuum (UHV) conditions. However, the grain boundaries in such graphene films act as scattering centers for electron waves, limiting electron mobility. ${ }^{\mathbf{4 1 , 4 2}}$ To minimize the grain boundary effect, I have modified the graphene growth process by supplying $\mathrm{CH}_{4}$ in short pulses ${ }^{31}$ instead of the conventional continuous $\mathrm{CH}_{4}$ flow during the CVD growth of graphene on $\mathrm{Cu}$, as illustrated in Fig. 1. The details of the synthesis process are provided in
Fig. S1 (see ESI $\dagger$ ). In the case of continuous $\mathrm{CH}_{4}$ flow used in literature so far, the rate of nucleation is very high, which leads to high density of graphene nucleation seeds over the $\mathrm{Cu}$ surface. ${ }^{43-47}$ These nucleation seeds interfere each other when growth persists, and hence, the graphene domains do not grow in the preferred orientation, and the average graphene domain size shrinks. On the other hand, in the pulsed-grown method, graphene domains are seen to coalesce in a preferred direction to form larger islands of $\sim 100 \mu \mathrm{m}$ size (shown by arrows in Fig. S2 of ESI $\dagger$ ). This provides evidence that the nucleation rate is expected to be slow in the pulsed-grown method, which leads to low density of graphene nucleation seeds. It can be noted that the preliminary annealing of the $\mathrm{Cu}$ substrate leads to a reconstruction of atomic distribution over the surface, ${ }^{48}$ which causes directional growth of graphene domains, but not coalescence. As the $\mathrm{Cu}$ substrates used in this work were not pre-annealed, the directional growth due to substrate effect can be ruled out.

In addition to lower nucleation rate, the purpose of the pulsed $\mathrm{CH}_{4}$ gas is to suppress carbon segregation at the defect sites in $\mathrm{Cu}$, which aids in removing the multilayer patches generally formed using continuous $\mathrm{CH}_{4}$ flow. ${ }^{43}$ Moreover, the restoration time after each $\mathrm{CH}_{4}$ pulse increases the catalytic activity of $\mathrm{H}_{2}$ gas, during which hydrogen etches off the amorphous carbon atoms and the $\mathrm{sp}^{3}$ carbon network present on the surface. Repeated alternating cycles of growth with $\mathrm{CH}_{4}$ pulse and restoration of $\mathrm{H}_{2}$ treatment promote the complete transformation of the carbon chain to a most stable $\mathrm{sp}^{2} \mathrm{C}-\mathrm{C}$ network,

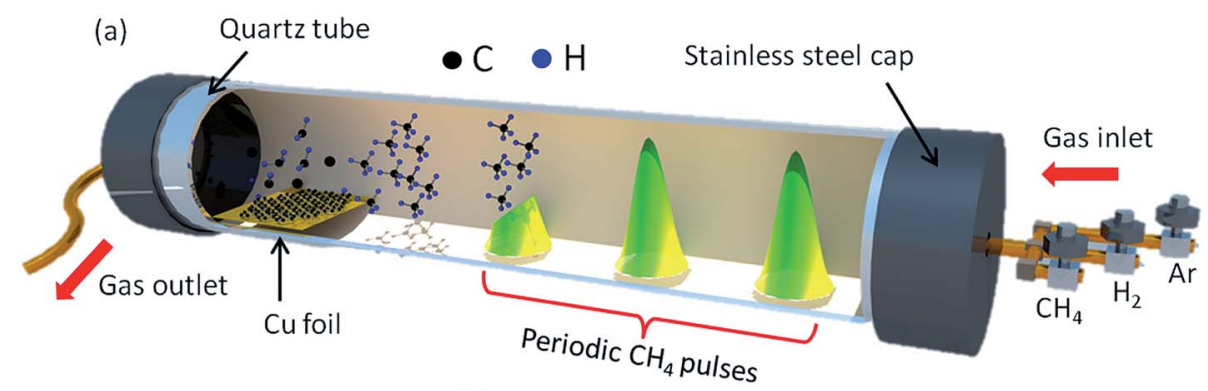

(b)

(c)

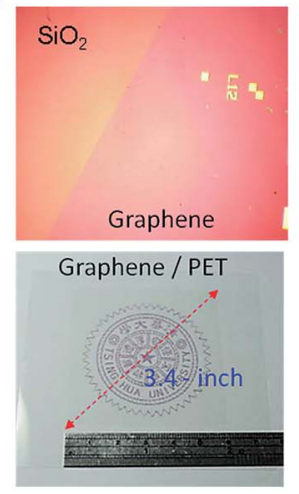

(d)

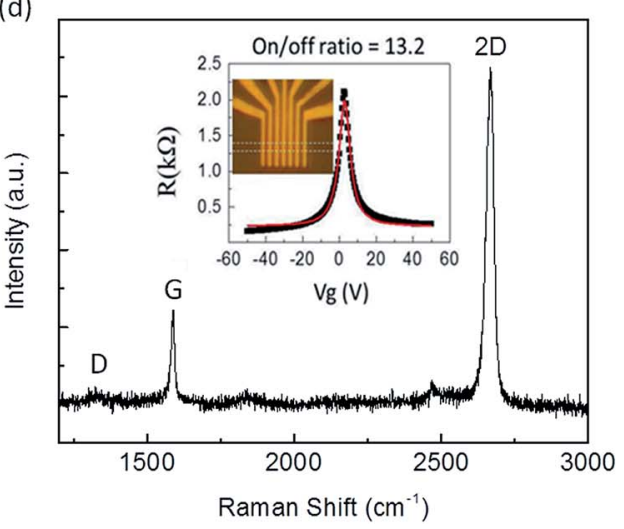

Fig. 1 (a) Schematic of the designed CVD system with introduction of periodic $\mathrm{CH}_{4}$ pulses into the reaction zone. (b) OM image of pulse-grown graphene film transferred onto marked $300 \mathrm{~nm} \mathrm{SiO} / 2$ Si substrate. The graphene film is clearly distinguishable from bare $\mathrm{SiO}_{2}$ with the color contrast. (c) Camera image of 3.4-inch graphene film transferred onto $125 \mu \mathrm{m}$ thick PET substrate. (d) Raman spectrum of transferred graphene film on $\mathrm{SiO}_{2}$ substrate. Both $\mathrm{G}$ and $2 \mathrm{D}$ band peaks are very symmetric with full width at half maximum (FWHM) of 15 and $30 \mathrm{~cm}^{-1}$, respectively. The $I_{2 D} / I_{G}$ intensity ratio is $>1.5$, which confirms that the prepared graphene is monolayer. A slight $D$ band intensity is present in the Raman spectrum, which probably arises from the etching process. The inset shows the $R$ vs. $V_{\mathrm{g}}$ plot for graphene-based FET in back-gate configuration. 
as shown in Fig. S3 (ESI†). These hydrogen atoms not only promote the activation of physisorbed methane but also remove defects in carbon structures, which can be described in the following equation,

$$
\mathrm{H}_{\mathrm{s}}+(\text { graphene-defects }) \Leftrightarrow(\text { graphene- } \mathrm{C})+\left(\mathrm{CH}_{x}\right)_{\mathrm{s}}
$$

As a consequence, structural defects such as pentagon and heptagon structures are minimized, leading to a clean and defect-free graphene surface. The schematic illustration in Fig. S3a (see ESI $\dagger$ ) shows the evolution of surface defects emerging from unexpected bumps, protrusions, and grooves on the surface; those bring up electron scattering issues in graphene, leading to Fermi velocity degradation. The dwell time of $\mathrm{CH}_{4}$ pulses also affects graphene domain size, as shown in Fig. S2. $\dagger$ Longer dwell time of $\mathrm{CH}_{4}$ pulses leads to a smaller graphene domain size of $\sim 2-3 \mu \mathrm{m}$ (Fig. S2a and $\mathrm{b} \dagger$ ), whereas larger domain sizes of $\sim 20-30 \mu \mathrm{m}$ could be achieved for shorter dwell time of $\mathrm{CH}_{4}$ pulses, as shown in Fig. S2c and d. $\dagger$ The preferred orientations of graphene domains are marked with arrows in Fig. S2, $\uparrow$ where these domains are merged together to form larger islands $(\sim 100 \mu \mathrm{m})$. The large graphene domain size along with the defect-free surface increases the mobility of the electrons, due to the low defect density and grain boundary effect, ${ }^{31}$ thereby decreasing the $R_{\mathrm{s}}$. The as-prepared graphene film was transferred onto $300 \mathrm{~nm} \mathrm{SiO} / \mathrm{Si}$ substrate using the conventional poly(methyl methacrylate) (PMMA)-based transfer process, as shown in Fig. S4 (see ESI $\dagger$ ), similar to Lin et al., ${ }^{49}$ and the optical microscope (OM) image is shown in Fig. 1b. For electromechanical characterization, the graphene film was also transferred onto a flexible polyethylene terephthalate (PET) substrate, and the corresponding OM image is shown in Fig. 1c. The quality of our graphene film is reflected from the symmetric $2 \mathrm{D}$ peak, large $I_{2 \mathrm{D}} / I_{\mathrm{G}}$ ratio $(>1.5)$ and absence of $\mathrm{D}$ peak measured from the Raman spectrum on $\mathrm{SiO}_{2} / \mathrm{Si}$ substrate, as shown in Fig. 1d, which confirm that the prepared graphene is predominantly monolayer and defect free. Moreover, the $R v s$. $V_{\mathrm{g}}$ plot of the graphene-based field effect transistor (FET), shown inset in Fig. 1d, shows strong electron-hole symmetry, a narrow profile width $(\sim 5.5 \mathrm{~V})$, and gate modulation (on/off ratio) of about 13.2, which are the characteristic features of high-quality graphene..$^{\mathbf{5 0 , 5 1}}$

Several methods have been perused in order to reduce the $R_{\mathrm{s}}$ of graphene, including doping with TFSA, post annealing, flattening and stacking of graphene films. The schematics of the above processes are mentioned in Fig. S5 (see ESI $\dagger$ ), and the overall processes are summarized below.

The organic dopant TFSA is hydrophobic in nature and an excellent candidate for doping graphene due to its following advantages: effective electron-withdrawing ability, high transmittance, smooth surface, and superior environmental stability. ${ }^{33}$ TFSA has been used as a p-type dopant in carbon nanotubes $^{52}$ and also in graphene. ${ }^{19,33,34,53}$ TFSA, with a $-\mathrm{NH}$ group, easily loses a proton and transforms to TFSI upon accepting an electron from graphene, which is characteristic of a Brønsted acid. ${ }^{54}$ When an electron is transferred from graphene to TFSA, a charge-transfer complex is formed according to eqn (2),

$$
\begin{aligned}
& \left(\mathrm{CF}_{3} \mathrm{SO}_{2}\right)_{2} \mathrm{NH}+\text { Graphene } \rightarrow \\
& {\left[\left(\mathrm{CF}_{3} \mathrm{SO}_{2}\right)_{2} \mathrm{~N}\right]^{-}+\mathrm{H}^{+}+\text {Graphene }^{+}}
\end{aligned}
$$

Thus, there is hole doping in the graphene that results in a shift of the Fermi level. In order to visualize this doping effect, I have performed electronic band structure calculation of TFSAdoped graphene using density functional theory (DFT), and the results are displayed in Fig. 2a and b. The details of DFT calculation are mentioned in the method section. Fig. 2a shows the optimized position of TFSA molecules absorbed on the surface of monolayer graphene. The constituent atoms of TFSA, such as N, S, O, C and F are marked in blue, yellow, red, grey and green, respectively. From the band structure result (Fig. 2b), the band gap value was found to be almost zero; i.e., the gap between conduction band minimum (CBM) and valence band maximum (VBM) at the high-symmetry $K$ point of the Brillouin zone and the Fermi level shifts below VBM, which is characteristic of p-doped graphene. ${ }^{55,56}$ TFSA-doped graphene was prepared by spinning a few droplets of TFSA over graphene film at $3000 \mathrm{rpm}$ for $30 \mathrm{~s}$. The Raman spectra of pure and TFSAdoped graphene are shown in Fig. 2c. The characteristic Raman peaks for pristine graphene, located at $1588 \mathrm{~cm}^{-1}$ and $2669 \mathrm{~cm}^{-1}$, correspond to $\mathrm{G}$ band (first-order Raman process) and $2 \mathrm{D}$ band (second-order Raman process), respectively. ${ }^{57}$ Both pristine and TFSA-doped graphene exhibit very intense and symmetric $2 \mathrm{D}$ peaks with a large $I_{2 \mathrm{D}} / I_{\mathrm{G}}$ ratio $(>1.5)$. Compared to pristine graphene, doped graphene shows a large blue shift for both $\mathrm{G}$ peak $\left(\Delta G=6 \mathrm{~cm}^{-1}\right)$ and $2 \mathrm{D}$ peak $\left(\Delta 2 D=33 \mathrm{~cm}^{-1}\right)$, which confirms that there is charge transfer from graphene to TFSA, thereby causing p-doping, ${ }^{58}$ which coincides with my calculation result. Interestingly, the D-peak, which corresponds to the TO phonons around the $K$ point of the Brillouin zone and is the signature of defects on the graphene surface, is found to be absent before or after doping. This observation indicates that TFSA treatment is not destructive to the chemical bonds of graphene, which is quite similar to $\mathrm{HNO}_{3}$-doped graphene. ${ }^{18}$

In the pursuit of transparent conducting applications, the optical and electrical properties of $1 \times 1 \mathrm{~cm}^{2}$ pristine and doped graphene films prepared by pulsed-grown method were analyzed, as shown in Fig. 3. The optical transmittance of the graphene films in the visible range was measured using an UVVIS-NIR spectrophotometer (UV-3600, Shimadzu). For this measurement, graphene films were transferred on a cover glass as a single layer and as multiple stacked layers, and a bare cover glass was used as a reference. Multiple-layered "graphene stacks" were created using the transfer process sequentially. Fig. 3a depicts the wavelength dependence of the optical transparency of one- to four-layer-stacked pristine graphene films. The transmittance, $T_{\mathrm{r}}(\%)$, of stacked graphene films at $\lambda$ $=550 \mathrm{~nm}$ extracted from (a) was found to be 97.3, 94.6, 92.9 and 90 for one, two, three and four layers, respectively (Fig. 3b). Because each graphene layer has an opacity of $2.3 \%,{ }^{8}$ the transmittance decreases by $\sim 2.3 \%$ for each added layer, indicating that the film behaves as a set of individual graphene layers. ${ }^{8,59}$ A slight variation in the transmittance values as per layer numbers predicted from theoretical calculation may be due to impurities present in the interface of the artificially built 
(a)

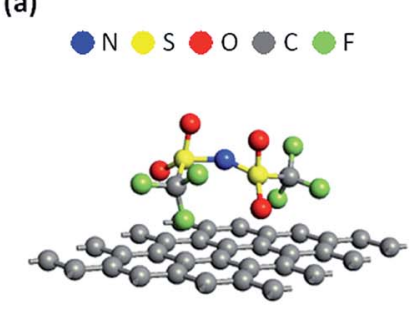

(c)

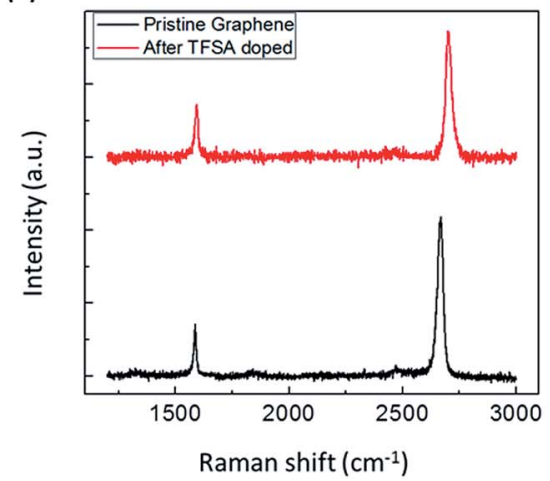

(b)
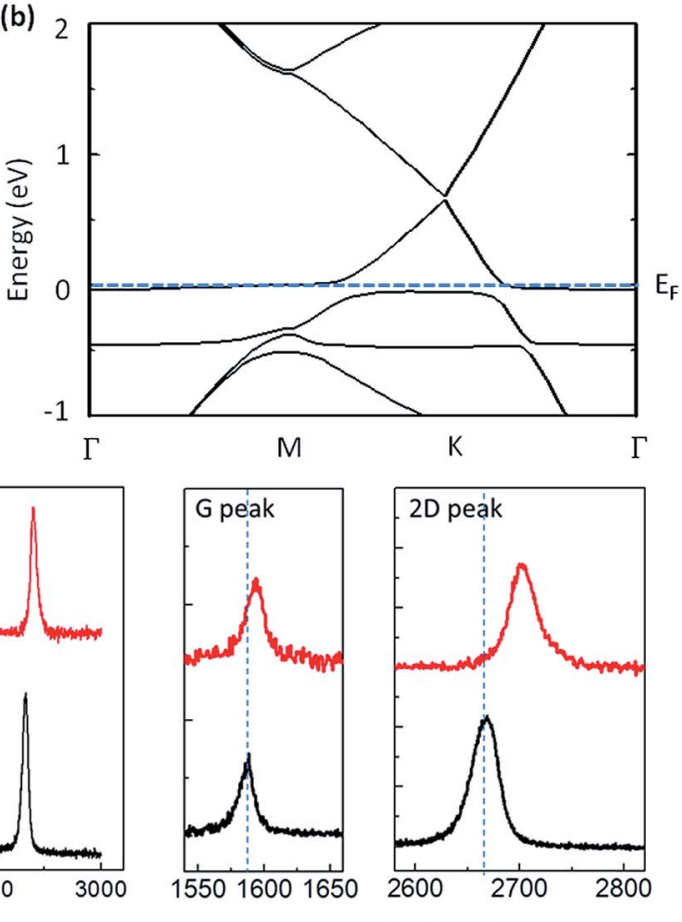

Raman shift $\left(\mathrm{cm}^{-1}\right)$

Fig. 2 Effect of TFSA doping on the Raman spectra of pulsed-grown graphene. (a) Optimized position of TFSA molecules absorbed on the surface of monolayer graphene. The constituent atoms of TFSA, such as N, S, O, C and F are marked in blue, yellow, red, grey and green, respectively. (b) Band structure calculation of TFSA-doped graphene using DFT. The band gap is almost zero; i.e., the gap between conduction band minimum and valence band maximum at the high symmetry $K$ point of the Brillouin zone and the Fermi level shifts below VBM due to $p$ doping. (c) Raman spectra of CVD graphene before and after TFSA doping. The G and 2D peaks of both the graphene films are extrapolated and plotted in separate panels for comparison. Compared to pristine graphene, doped graphene shows a large blue shift for both $G$ peak $(\Delta G=$ $\left.6 \mathrm{~cm}^{-1}\right)$ and $2 \mathrm{D}$ peak $\left(\Delta 2 D=33 \mathrm{~cm}^{-1}\right)$, which confirms that there is charge transfer from graphene to TFSA.

weak van der Waals stacked layers. ${ }^{60}$ Similar behavior has also been observed by Karsy et al. ${ }^{7}$ for stacked graphene layers. The evolution of graphene layers is also detectable from the change in color contrast observed from the optical images, as shown in the inset of Fig. $3 \mathrm{~b}$, which is minimum for one layer and maximum for four layers. The optical transmittance of TFSAdoped graphene also varies in a similar fashion to pristine graphene. For comparison, the optical transmittance of pristine and doped monolayer graphene is shown in Fig. 3c. It is observed that the $T_{\mathrm{r}}$ values of both films at $\lambda=550 \mathrm{~nm}$ are almost same, and hence, TFSA doping has no effect on the $T_{\mathrm{r}}$ of the graphene film. Fig. 3d shows the $R_{\mathrm{S}}$ of pristine and doped graphene films measured using van der Pauw method. The details of $R_{\mathrm{s}}$ measurement of graphene films are shown in Fig. S6 (see ESI $\dagger$ ). In both the cases, $R_{\mathrm{s}}$ decreases with the increase in number of graphene layers. The $R_{\mathrm{s}}$ of monolayer graphene film is $288 \Omega \mathrm{sq}^{-1}$ with $T_{\mathrm{r}}$ of $97.3 \%$, whereas that of four-layer graphene is $86 \Omega \mathrm{sq}^{-1}$, while still maintaining a relatively high $T_{\mathrm{r}}$ of about $90 \%$. Similarly, the $R_{\mathrm{S}}$ of doped graphene varies from $134 \Omega \mathrm{sq}^{-1}$ to a lowest value of $40 \Omega \mathrm{sq}^{-1}$ with the increase in number of graphene layers from one to four, while maintaining the same $T_{\mathrm{r}}$ values as those of pure graphene layers. In principle, the $R_{\mathrm{S}}$ of multilayer film, $R_{\mathrm{s} n}$, is related to the $R_{\mathrm{S}}$ of one layer film, $R_{\mathrm{s} 1}$, by the relation $R_{\mathrm{S} n}=R_{\mathrm{S} 1} / n$, where $n$ is the number of graphene layers if all layers are acting independently of each other. However, the average $R_{\mathrm{S}}$ of our films decreases slightly by stacking four graphene layers. The discrepancy might be explained by considering that the cracks in one film are bridged by its neighboring films, thus increasing the conductivity. ${ }^{61}$ As stated earlier, when an electron is transferred from the graphene to TFSA, graphene becomes p-doped according to eqn (2). This results in a shift of the Fermi level (Fig. 3b), which increases the carrier concentration and thus the conductivity of the graphene layers. With the increase in layer numbers, there is further increment in the carrier concentration and density of states (DOS) at the Dirac point, which results in higher conductivity. This effect was first observed with singlewalled carbon nanotubes (SWCNTs), where the doping of the SWCNTs with TFSA led to an increase in the conductivity of SWCNT films. ${ }^{52}$ Since SWCNTs and graphene have essentially the same chemical structure, it is worth noting that TFSA doping yields lower sheet resistance.

Fig. 4a compares the sheet resistance of our graphene films prepared by pulsed-grown method with some others reported in literature as a function of $T_{\mathrm{r}}$ at $\lambda=550 \mathrm{~nm}$. An extremely low $R_{\mathrm{s}}$ of $40 \Omega \mathrm{sq}^{-1}$ was observed for TFSA-doped four-layer stacked graphene films at a $T_{\mathrm{r}}$ of $90 \%$. Such a low value of $R_{\mathrm{S}}$ is promising for designing an extremely efficient transparent conductor, and the $T_{\mathrm{r}}$ value is almost comparable with that of ITO. ${ }^{62,63}$ The $R_{\mathrm{S}}$ of our pristine and TFSA-doped graphene films 
(a)

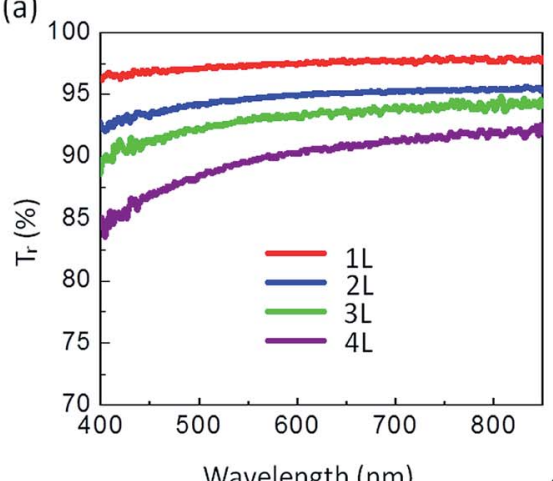

(c)

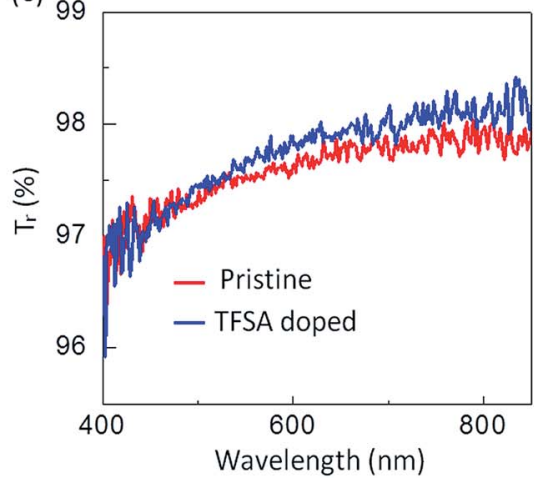

(b)

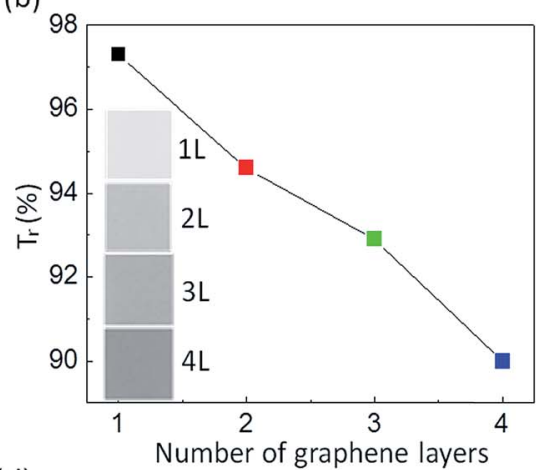

(d)

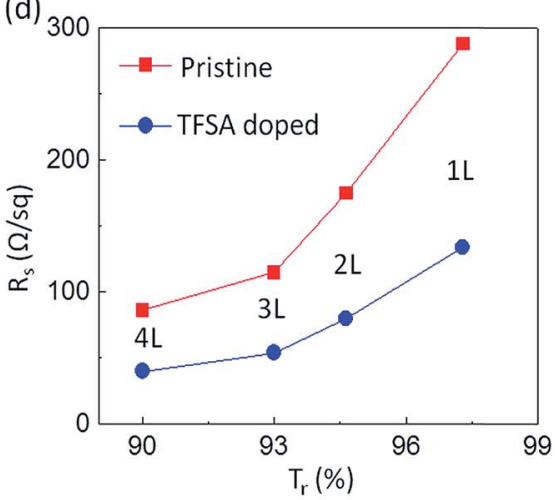

Fig. 3 Evaluation of pulsed-grown graphene films as transparent conductor. (a) Transmittance spectra of one-to four-layer stacked graphene films in the visible region of the energy spectrum. (b) $T_{r}(\%)$ as a function of the number of graphene layers extracted from (a) at $\lambda=550 \mathrm{~nm}$. Inset shows photographs of stacked graphene layers. (c) Comparison of transmittance spectra between one-layer pristine graphene and TFSA-doped graphene. (d) $R_{\mathrm{s}} v s$. $T_{\mathrm{r}}(\%)$ of pristine and TFSA-doped graphene films for one to four stacked layers.

is very low compared to that of wet transferred graphene ${ }^{61}$ and $\mathrm{HNO}_{3}$-doped graphene. ${ }^{60}$ However, these values are a little higher than the theoretical calculated values. ${ }^{64}$ These higher experimental $R_{\mathrm{S}}$ values could be due to the presence of small metallic contaminants on the graphene surface during the transfer process. For the monolayer case, the $R_{\mathrm{S}}$ of prepared graphene films is also low compared to $\mathrm{CHCl}_{3}$-doped graphene $^{24}$ and annealed and polished graphene, ${ }^{65}$ but a little higher than KI-doped graphene. ${ }^{66}$

Besides TFSA doping $(T)$, I carried out post annealing $(A)$ and flattening $(F)$ of graphene films to reduce the $R_{\mathrm{S}}$, as shown in Fig. $4 \mathrm{~b}$. The maximum reduction in the $R_{\mathrm{S}}\left(\Delta R_{\mathrm{s}}\right)$ was observed for $T(68 \%)$ compared to $A(66 \%)$ and $F(22 \%)$. Interestingly, the combination of all three effects $(T+A+F)$ shows a maximum $\Delta R_{\mathrm{S}}$ of $80 \%$, whereas other combinations $(A+T),(F+T)$ and $(A+$ $F)$ show $\Delta R_{\mathrm{S}}$ of $79 \%, 70 \%$ and $64 \%$, respectively. The above methods also have been applied to graphene prepared by continuous $\mathrm{CH}_{4}$ flow in literature, including TFSA doping, ${ }^{19,33,67}$ annealing, ${ }^{35,36}$ and stacking,,${ }^{7,23,24}$ as shown in ESI Table S1. $\dagger$ The reduction is $R_{\mathrm{S}}$ value is $30-75 \%, 7-30 \%$ and $50-58 \%$ for TFSA doping, annealing and stacking, respectively, which are comparatively less with reference to pulsed-grown graphene. During post annealing, the residual PMMA present on the surface of graphene decomposes at a temperature $>160{ }^{\circ} \mathrm{C} .{ }^{49} \mathrm{It}$ is expected that the decomposition products of this molecule form cross links with the graphene surface by forming dangling bonds, and the surface absorbs $\mathrm{O}_{2}$ or water vapor during cooling to room temperature, becoming heavily p-doped, which leads to increased conductance. Flattening of graphene films was carried out using PMMA, in which the PMMA/graphene stack was prepared and heated at elevated temperatures ( 150-160 $\left.{ }^{\circ} \mathrm{C}\right)$. Heating of PMMA resulted in softening and expanding PMMA, allowing it to relax flat upon the substrate and thereby minimizing any wrinkling of the graphene, in turn restricting mechanical and electrical degradation, as reported by Goniszewski et al. ${ }^{37}$ However, the effect of flattening was found to be very insignificant compared to TFSA doping and post annealing (Fig. 4b).

In addition to high optical $T_{\mathrm{r}}$ and low $R_{\mathrm{s}}$, the graphene film should show excellent mechanical flexibility when used to make flexible and stretchable electrodes. ${ }^{68}$ In order to investigate the mechanical flexibility of the present graphene films, the pristine as well as the doped monolayer graphene films were transferred onto a $125 \mu \mathrm{m}$ thick polyethylene terephthalate (PET) substrate coated with a thin PDMS layer (Nan Ya Plastics Corporation, Taiwan (BH216)). Prior to graphene transfer, $30 \mathrm{~nm} \mathrm{SiO}_{2}$ was deposited on PET by E-gun evaporation technique to reduce its surface roughness. Fig. 5a shows the photograph of highly transparent graphene film transferred onto flexible PET substrate. To check the environmental stability of TFSA doping, the $R_{\mathrm{S}}$ of TFSA-doped graphene was measured for several days (Fig. 5b). Interestingly, there was no deterioration in $R_{\mathrm{s}}$ observed, even after 18 days, endorsing its superior environmental stability. Fig. $5 \mathrm{c}$ presents the $R_{\mathrm{s}}$ of pristine graphene film 
(a)

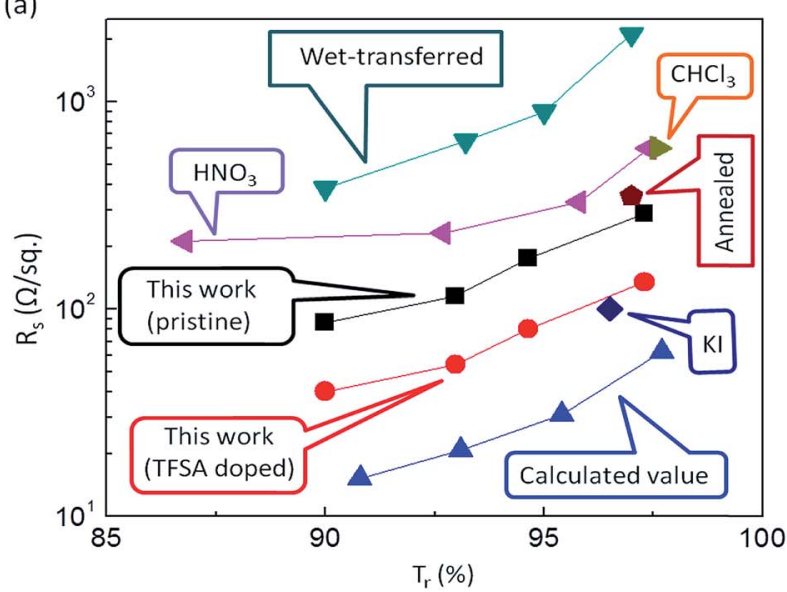

(b)

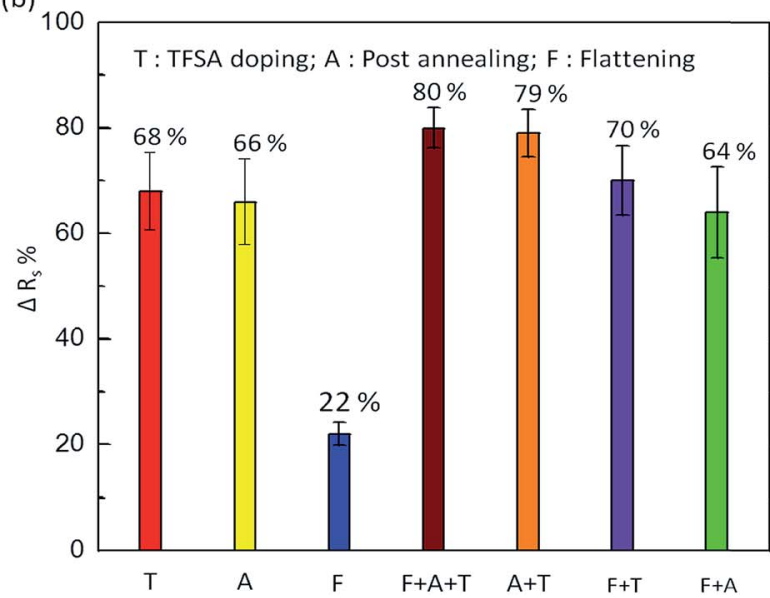

Fig. 4 (a) Comparison of $R_{\mathrm{s}}$ of the pulsed-grown graphene films with those reported in the literature as a function of $T_{\mathrm{r}}(\%)$ at $\lambda=550 \mathrm{~nm}$. An extremely low $R_{\mathrm{s}}$ of $40 \Omega \mathrm{sq}^{-1}$ was observed for TFSA-doped fourlayer stacked graphene films at a $T_{r}$ of $90 \%$, which is almost comparable with that of ITO. ${ }^{62,63}$ The $R_{\mathrm{s}}$ values of pristine (black squares) and TFSA-doped graphene films (red circles) of the present work are very low compared to those of wet transferred graphene (blue-green triangles) ${ }^{61}$ and $\mathrm{HNO}_{3}$ doped graphene (purple triangles). ${ }^{60}$ However, these values are slightly higher than the theoretical calculated values. ${ }^{64}$ For the monolayer case, the $R_{\mathrm{s}}$ of the present graphene films is also low compared to $\mathrm{CHCl}_{3}$-doped graphene (olive-green triangle) ${ }^{24}$ and annealed graphene (chocolate-brown pentagon), ${ }^{65}$ but slightly higher than $\mathrm{KI}$-doped graphene (blue diamond). ${ }^{66}(\mathrm{~b})$ Reduction in $R_{\mathrm{s}}\left(\Delta R_{\mathrm{s}}\right)$ of graphene films by TFSA doping $(T)$, post annealing $(A)$, flattening $(F)$ and some combination of these processes. The maximum $\Delta R_{\mathrm{s}}$ is $68 \%$ for TFSA doping compared to post annealing $(66 \%)$ and flattening (22\%). The combination of all three effects $(T+A+F)$ shows maximum $\Delta R_{\mathrm{s}}$ of $80 \%$, whereas other combinations $(A+T),(F+T)$ and $(A+F)$ show $\Delta R_{\mathrm{s}}$ of 79,70 and $64 \%$, respectively.

for bending-recovery cycles. For each cycle, graphene/PET was first bent to an angle of $162^{\circ}$ (radius of curvature $=0.35 \mathrm{~mm}$ ) with an interval of $18^{\circ}$ and then fully relaxed in the subsequent interval. The schematic diagram of graphene/PET bending is shown in Fig. S7 (see ESI $\dagger$ ). The arrows indicate the direction of bending and recovery. Prior to bending, the $R_{\mathrm{S}}$ of pristine graphene/PET was $419 \Omega \mathrm{sq}^{-1}$, which is higher than the $288 \Omega$ $\mathrm{sq}^{-1}$ observed for graphene/ $/ \mathrm{SiO}_{2}$, and this is probably due to the high RMS surface roughness of PET ( $4.3 \mathrm{~nm}$ ) compared to pure $\mathrm{SiO}_{2}(1 \mathrm{~nm})$. During bending, there was slight increase in the $R_{\mathrm{s}}$, then it recovered after releasing the stress. This process was performed through the repeated bending and relaxing steps up to 100 cycles (Fig. 5d). Interestingly, the $R_{\mathrm{s}}$ remained unperturbed up to 100 bending cycles, which demonstrates the high flexibility of the prepared graphene film, similar to those observed by other groups. ${ }^{\mathbf{9 9 , 7 0}}$

Bending of PET substrate produced surface strain on the graphene/PET film. The tensile strain was determined by the relatively extended length of graphene film upon bending of the underlying supporting PET substrate. ${ }^{71}$ By assuming the length of the neutral axis of flat PET to be $L_{0}$, which changes with respect to the subsequent bending angle $(\theta)$, the bending radius $(r)$ was calculated using $L_{0}=r \times \theta$. Furthermore, the uniaxial tensile strain was calculated from the following relation, ${ }^{72,73}$

$$
\varepsilon=\frac{100 t}{(2 r+t)} \%
$$

where $t$ is the thickness of the PET substrate. Fig. 4c inset shows the relative change in $R_{\mathrm{s}}$ versus strain due to bending. At a maximum tensile strain of $1.77 \%$, the $R_{\mathrm{s}}$ of the graphene film increased by $10-11 \%$, which is much smaller than the $321 \%$ observed for ITO at $2.14 \%$ tensile strain. ${ }^{74}$ Such a minor change in the $R_{\mathrm{S}}$ implies that the prepared graphene films are much more robust against bending and have advantages over ITO films in terms of superior mechanical flexibility.

\section{Experimental}

\section{Modified graphene growth process}

Graphene sheets used in the current study were grown by atmospheric pressure chemical vapor deposition (APCVD) on polycrystalline $\mathrm{Cu}$ foils, using methane (99.99\%) as carbon source. Prior to growth, the $\mathrm{Cu}$ foils were cleaned with acetone and isopropyl alcohol (IPA) by sonication, followed by etching in acetic acid for $30 \mathrm{~min}$ to remove surface oxides. Then, the $\mathrm{Cu}$ foils were mounted in the CVD chamber, and graphene growth process was configured in several steps in the presence of steady flow of hydrogen and argon gas in the proportion of 10-20:300 sccm. The schematic of the growth process is shown in Fig. S1 (see $\mathrm{ESI}^{\dagger}$ ). In the first step, the furnace was ramped up to $1050{ }^{\circ} \mathrm{C}$ over $40 \mathrm{~min}$. In the second step, pre-annealing was carried out by holding the temperature at $1050{ }^{\circ} \mathrm{C}$ for $30 \mathrm{~min}$. By this step, the oxide on the copper foil surface was being removed and the internal stress was released by recrystallization to make the surface of copper foil smoother. Then, methane with flow rate of $<1 \mathrm{sccm}$ was fed into the reaction chamber for 50 min (15-25 cycles) in step 3, during which graphene growth occurred. In each cycle, $\mathrm{CH}_{4}$ pulse was given for $30 \mathrm{~s}$ and then released for $1 \mathrm{~min}$. This process was carried out by means of a control device, which controlled the import flow rate of the $\mathrm{CH}_{4}$ gas by controlling the opening and closing of the gas valve. That is to say, the $\mathrm{CH}_{4}$ supply during this reaction step was changed continuously (first increased and then decreased in one cycle), which is different from the constant gas supply process in prior literature. Finally, the $\mathrm{Cu}$ 
(a)

(c)
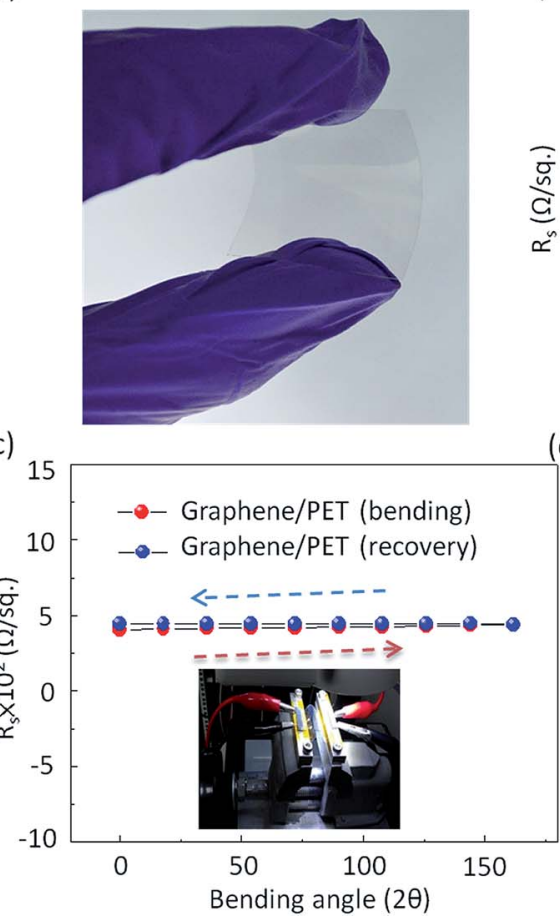

(b)

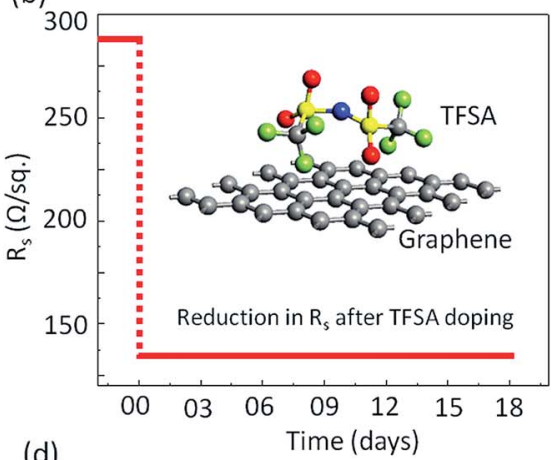

(d)

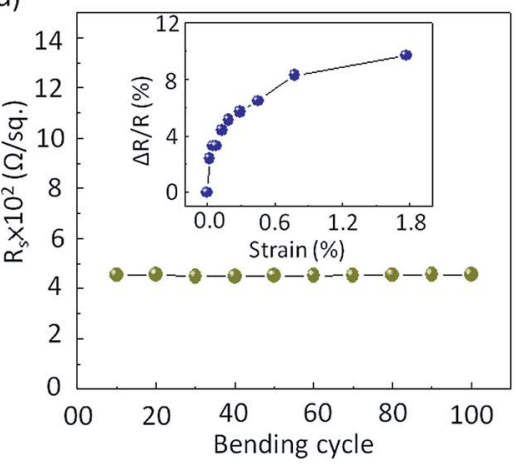

Fig. 5 Analysis of electromechanical properties of graphene films. (a) Photograph showing highly transparent graphene film transferred onto flexible PET substrate. (b) Reduction in $R_{\mathrm{s}}$ of graphene film after TFSA doping and its environmental stability test. The $R_{\mathrm{s}}$ remains preserved up to 18 days, revealing superior environmental stability. (c) The $R_{\mathrm{s}} v \mathrm{~s}$. bending angle of pristine graphene measured on PET substrate under different bending conditions. The arrows show the direction of bending and recovery. Inset shows a photograph of the mechanical flexibility test setup. (d) The $R_{\mathrm{s}} v \mathrm{~s}$. bending cycles for single-layer graphene/PET substrate. The $R_{\mathrm{s}}$ remains unperturbed up to 100 bending cycles. Inset shows the relative change in $R_{\mathrm{s}}$ versus strain due to bending. At a maximum tensile strain of $1.77 \%$, the $R_{\mathrm{s}}$ of the graphene film increased by $10-11 \%$ only.

foils were moved to the cooling zone, which is equipped with a cooling system, in step 4 .

\section{Graphene transfer process using PMMA}

Graphene layers on $\mathrm{Cu}$ foils were coated with $300 \mathrm{~nm}$ thick PMMA, followed by etching in a $16.5 \%$ aqueous hydrochloric acid solution. The PMMA film, along with the attached graphene, was detached from the metal substrate after a whole night. The PMMA/graphene film was then rinsed with DI water, transferred to a silicon or target substrate, and rinsed with warm acetone to remove the PMMA. Photographs of the graphene transfer process are shown in Fig. S4 (see ESI $\dagger$ ).

\section{Experimental methods to reduce sheet resistance}

TFSA-doped graphene was prepared by spinning a few droplets of TFSA over graphene film at $3000 \mathrm{rpm}$ for $30 \mathrm{~s}$. Post annealing was carried out in two steps, first at $250{ }^{\circ} \mathrm{C}$ in air and then at $250^{\circ} \mathrm{C}$ in the presence of mixed $\mathrm{H}_{2}(200 \mathrm{sccm})$ and $\mathrm{Ar}(400 \mathrm{sccm})$ atmosphere for $2 \mathrm{~h}$, similar to Lin et al..$^{49}$ Flattening of graphene film was performed by spin coating of PMMA at $3000 \mathrm{rpm}$ for $30 \mathrm{~s}$, followed by heating at elevated temperatures $(\sim 150-160$ ${ }^{\circ} \mathrm{C}$ ) and, finally, removing PMMA using warm acetone. Stacked graphene films were prepared by sequential transfer of one graphene layer over another, up to four layers. No adhesive was used at the interfaces, and the interlayer bonding is only due to van der Waals force.

\section{Band structure calculation of TFSA-doped graphene}

Band structure calculation was performed by placing the TFSA molecule on the top of single-layer graphene. The pseudopotential density functional SIESTA package was used for band structure calculations, ${ }^{75,76}$ with the local density approximation (LDA) representing the exchange correlation potential ${ }^{77,78}$ and with an energy mesh cutoff of 150 Ry. Optimization of initial experimental structures was performed using analytical energy gradient with respect to atomic coordinates and unit cell parameters. The structures were relaxed until the force on each atom was less than $0.01 \mathrm{eV}^{-1}$. The Brillouin zone was sampled with a grid of $5 \times 5 \times 1 \mathrm{k}$-points within the Monkhorst-Pack scheme. ${ }^{79}$ Band structures were calculated along the high symmetry points using the path $\Gamma-M-K-\Gamma$.

\section{Conclusions}

In summary, high-quality graphene was prepared by supplying methane pulses instead of the conventional continuous methane flow used in CVD. The lowest sheet resistance of a few hundred $\Omega \mathrm{sq}^{-1}$, the on/off ratio of $\sim 13$ for the graphene-based FET, and the high $I_{2 \mathrm{D}} / I_{\mathrm{G}}$ ratio of $>1.5$ demonstrate the quantitative signatures of graphene quality. Several processes, including TFSA doping, post annealing, flattening and stacking of graphene films, were carried out in order to reduce the sheet resistance, making them suitable for application as transparent 
conducting electrode. An extremely low sheet resistance of $40 \Omega$ $\mathrm{sq}^{-1}$ for TFSA-doped four-layer stacked graphene films at a transmittance of $90 \%$ was reported, which is almost comparable with that of ITO. TFSA doping was found to be very effective for increasing the conductivity of graphene film. Interestingly, the combination of TFSA doping, post annealing and flattening of graphene films was found to reduce the sheet resistance by $80 \%$, which is the highest value reported so far. Besides, the superior mechanical flexibility of this highly conducting and transparent film was also demonstrated, making it suitable as a transparent conducting electrode. This work provides a new pathway to obtain a suitable transparent conducting electrode for applications in various optoelectronic devices such as solar cells, touch screens, and transparent displays in the future.

\section{Conflicts of interest}

There are no conflicts to declare.

\section{Acknowledgements}

The author would like to acknowledge the financial support from the Department of Science and Technology, India, with sanction order No. SERB/F/5379/2017-2018 under Ramanujan Fellowship. The author also acknowledges Chao-Hui Yeh of National Tsing Hua University, Taiwan, for graphene transfer as well as useful discussion.

\section{Notes and references}

1 A. Kumar and C. Zhou, ACS Nano, 2010, 4, 11-14.

2 D. S. Hecht, L. Hu and G. Irvin, Adv. Mater., 2011, 23, 14821513.

3 J. Du, S. Pei, L. Ma and H. M. Cheng, Adv. Mater., 2014, 26, 1958-1991.

4 J. Ning, L. Hao, M. Jin, X. Qiu, Y. Shen, J. Liang, X. Zhang, B. Wang, X. Li and L. Zhi, Adv. Mater., 2017, 29, 1605028.

5 H. Kim, J. S. Horwitz, G. Kushto, A. Piqué, Z. H. Kafafi, C. M. Gilmore and D. B. J. Chrisey, J. Appl. Phys., 2000, 88, 6021-6025.

6 L. G. De Arco, Y. Zhang, C. W. Schlenker, K. Ryu, M. E. Thompson and C. Zhou, ACS Nano, 2010, 4, 2865-2873.

7 A. Kasry, M. A. Kuroda, G. J. Martyna, G. S. Tulevski and A. A. Bol, ACS Nano, 2010, 4, 3839-3844.

8 R. R. Nair, P. Blake, A. N. Grigorenko, K. S. Novoselov, T. J. Booth, T. Stauber, N. M. R. Peres and A. K. Geim, Science, 2008, 320, 1308.

9 X. Huang, T. Leng, M. Zhu, X. Zhang, J. Chen, K. Chang, M. Aqeeli, A. K. Geim, K. S. Novoselov and Z. Hu, Sci. Rep., 2015, 5, 18298.

10 S. Chen, L. Brown, M. Levendorf, W. Cai, S. Y. Ju, J. Edgeworth, X. Li, C. W. Magnuson, A. Velamakanni, R. D. Piner, J. Kang, J. Park and R. S. Ruoff, ACS Nano, 2011, 5, 1321-1327.

11 P. K. Nayak, C. J. Hsu, S. C. Wang, J. C. Sung and J. L. Huang, Thin Solid Films, 2013, 529, 312-316.
12 K. S. Kim, Y. Zhao, H. Jang, S. Y. Lee, J. M. Kim, K. S. Kim, J. H. Ahn, P. Kim, J. Y. Choi and B. H. Hong, Nature, 2009, 457, 706-710.

13 J. B. Wu, M. Agrawal, H. A. Becerril, Z. N. Bao, Z. F. Liu, Y. S. Chen and P. Peumans, ACS Nano, 2010, 4, 43-48.

14 T. Kobayashi, M. Bando, N. Kimura, K. Shimizu, K. Kadono, N. Umezu, K. Miyahara, S. Hayazaki, S. Nagai, Y. Mizuguchi, Y. Murakami and D. Hobara, Appl. Phys. Lett., 2013, 102, 023112 .

15 A. Ford, Graphene for Transparent Conductive Film Applications, LAP LAMBERT Academic Publishing, 2016.

16 P. P. Edwards, A. Porch, M. O. Jones, D. V. Morgan and R. M. Perks, Dalton Trans., 2004, 2995-3002.

17 K. K. Kim, A. Reina, Y. Shi, H. Park, L. J. Li, Y. H. Lee and J. Kong, J. Nanotechnol., 2010, 21, 285205.

18 S. Bae, H. Kim, Y. Lee, X. Xu, J. S. Park, Y. Zheng, J. Balakrishnan, T. Lei, H. R. Kim, Y. I. Song, Y. J. Kim, K. S. Kim, B. Özyilmaz, J. H. Ahn, B. H. Hong and S. Iijima, Nat. Nanotechnol., 2010, 5, 574-578.

19 X. Miao, S. Tongay, M. K. Petterson, K. Berke, A. G. Rinzler, B. R. Appleton and A. F. Hebard, Nano Lett., 2012, 12, 27452750.

20 J. O. Hwang, J. S. Park, D. S. Choi, J. Y. Kim, S. H. Lee, K. E. Lee, Y. H. Kim, M. H. Song, S. Yoo and S. O. Kim, ACS Nano, 2012, 6, 159-167.

21 L. D. Arsié, S. Esconjauregui, R. S. Weatherup, X. Wu, W. E. Arter, H. Sugime, C. Cepek and J. Robertson, RSC Adv., 2016, 6, 113185-113192.

22 M. Kim, K. J. Kim, S. J. Lee, H. M. Kim, S. Y. Cho, M. S. Kim, S. H. Kim and K. B. Kim, ACS Appl. Mater. Interfaces, 2017, 9, 701-709.

23 Y. Xu, H. Yu, C. Wang, J. Cao, Y. Chen, Z. Ma, Y. You, J. Wan, X. Fang and X. Chen, Nanoscale Res. Lett., 2017, 12, 254.

24 J. S. Choi, H. Choi, K. C. Kim, H. Y. Jeong, Y. J. Yu, J. T. Kim, J. S. Kim, J. W. Shin, H. Cho and C. G. Choi, Sci. Rep., 2016, 6, 24525.

25 B. Chandra, A. Afzali, N. Khare, M. M. El-Ashry and G. S. Tulevski, Chem. Mater., 2010, 22, 5179-5183.

26 C. Yan, K. S. Kim, S. K. Lee, S. H. Bae, B. H. Hong, J. H. Kim, J. H. Kim, H. J. Lee and J. H. Ahn, ACS Nano, 2012, 6, 20962103.

27 F. Schedin, A. K. Geim, S. V. Morozov, E. W. Hill, B. Blake, M. I. Katsnelson and K. S. Novoselov, Nat. Mater., 2007, 6, 652-655.

28 K. Rakhimov, A. Chaves and P. Lambin, Appl. Sci., 2016, 6, 256-266.

29 A. Capasso, T. Dikonimos, F. Sarto, A. Tamburrano, G. De Bellis, M. S. Sarto, G. Faggio, A. Malara, G. Messina and N. Lisi, Beilstein J. Nanotechnol., 2015, 6, 2028-2038.

30 G. H. Kim and S. Jeong, Sci. Rep., 2015, 5, 11227.

31 Z. Han, A. Kimouche, D. Kalita, A. Allain, H. A. Tash, A. R. Plantey, L. Marty, S. Pairis, V. Reita, N. Bendiab, J. Coraux and V. Bouchiat, Adv. Funct. Mater., 2014, 24, 964-970.

32 Y. C. Lai, B. S. Wu, S. C. Yu, P. Yu and G. C. Chi, IEEE Conf., 2013, 2436-2438. 
33 S. Tongay, K. Berke, M. Lemaitre, Z. Nasrollahi, D. B. Tanner, A. F. Hebard and B. R. Appleton, Nanotechnology, 2011, 22(16), 425701.

34 F. Bausi, A. Schlierf, E. Treossi, M. G. Schwab, V. Palermo and F. Cacialli, Org. Electron., 2015, 18, 53-60.

35 O. V. Tolochko, T. V. Larionova, T. S. Koltsova, M. V. Kozlova, E. V. Bobrynina, O. A. Martynova and V. E. Gasumyants, J. Phys.: Conf. Ser., 2017, 816, 012012.

36 W. Choi, Y. S. Seo, J. Y. Park, K. B. Kim, J. Jung, N. Lee and Y. Seo, IEEE Trans. Nanotechnol., 2015, 14, 70-74.

37 S. Goniszewski, J. Gallop, M. Adabi, K. Gajewski, E. Shaforost, N. Klein, A. Sierakowski, J. Chen, Y. Chen, T. Gotszalk and L. Hao, IET Circuits, Devices Syst., 2015, 9, 420-427.

38 X. Li, W. Cai, J. An, S. Kim, J. Nah, D. Yang, R. Piner, A. Velamakanni, I. Jung, E. Tutuc, S. K. Banerjee, L. Colombo and R. S. Ruoff, Science, 2009, 324, 1312.

39 Y. C. Lin, C. H. Jin, J. C. Lee, S. F. Jen, K. Suenaga and P. W. Chiu, ACS Nano, 2011, 5, 2362-2368.

40 X. Li, L. Colombo and R. S. Ruoff, Adv. Mater., 2016, 28, 6247-6252.

41 H. S. Song, S. L. Li, H. Miyazaki, S. Sato, K. Hayashi, A. Yamada, N. Yokoyama and K. Tsukagoshi, Sci. Rep., 2012, 2, 337.

42 V. Kochat, C. S. Tiwary, T. Biswas, G. Ramalingam, K. Hsieh, K. Chattopadhyay, S. Raghavan, M. Jain and A. Ghosh, Nano Lett., 2016, 16, 562-567.

43 G. H. Han, F. Günes, J. J. Bae, E. S. Kim, S. J. Chae, H. J. Shin, J. Y. Choi, D. Pribat and Y. H. Lee, Nano Lett., 2011, 11, 41444148.

44 Y. Ogawa, K. Komatsu, K. Kawahara, M. Tsuji, K. Tsukagoshi and H. Ago, Nanoscale, 2014, 6, 7288-7294.

45 H. Zhou, W. J. Yu, L. Liu, R. Cheng, Y. Chen, X. Huang, Y. Liu, Y. Wang, Y. Huang and X. Duan, Nat. Commun., 2013, 4, 2096.

46 L. P. Biŕo and P. Lambin, New J. Phys., 2013, 15, 035024.

47 X. Chen, L. Zhang and S. Chen, Synth. Met., 2015, 210, 95108.

48 I. V. Antonova, Phys.-Usp., 2013, 183, 1115-1122.

49 Y. C. Lin, C. C. Lu, C. H. Yeh, C. Jin, K. Suenaga and P. W. Chiu, Nano Lett., 2012, 12, 414-419.

50 T. J. Ha, J. Lee, D. Akinwande and A. Dodabalapur, IEEE Electron Device Lett., 2013, 34, 559-561.

51 J. W. Suk, W. H. Lee, J. Lee, H. Chou, R. D. Piner, Y. Hao, D. Akinwande and R. S. Ruoff, Nano Lett., 2013, 13, 14621467.

52 S. M. Kim, Y. W. Jo, K. K. Kim, D. L. Duong, H. J. Shin, J. H. Han, J. Y. Choi, J. Kong and Y. H. Lee, ACS Nano, 2010, 4, 6998-7004.

53 D. Kim, D. Lee, Y. Lee and D. Y. Jeon, Adv. Funct. Mater., 2013, 23, 5049-5055.

54 M. A. B. H. Susan, A. Noda, S. Mitsushima and M. Watanabe, Chem. Commun., 2003, 938-939.

55 M. Pykal, P. Jurečka, F. Karlický and M. Otyepka, Phys. Chem. Chem. Phys., 2016, 18, 6351-6372.
56 B. J. Schultz, R. V. Dennis, V. Lee and S. Banerjee, Nanoscale, 2014, 6, 3444-3466.

57 A. C. Ferrari, J. C. Meyer, V. Scardaci, C. Casiraghi, M. Lazzeri, F. Mauri, S. Piscanec, D. Jiang, K. S. Novoselov, S. Roth and A. K. Geim, Phys. Rev. Lett., 2006, 97, 187401.

58 R. Parret, M. Paillet, J. R. Huntzinger, D. Nakabayashi, T. Michel, A. Tiberj, J. L. Sauvajol and A. A. Zahab, ACS Nano, 2013, 7, 165-173.

59 P. Blake, P. D. Brimicombe, R. R. Nair, T. J. Booth, D. Jiang, F. Schedin, L. A. Ponomarenko, S. V. Morozov, H. F. Gleeson, E. W. Hill, A. K. Geim and K. S. Novoselov, Nano Lett., 2008, 8, 1704-1708.

60 G. Gao, W. Gao, E. Cannuccia, J. T. Tijerina, L. Balicas, A. Mathkar, T. N. Narayanan, Z. Liu, B. K. Gupta, J. Peng, Y. Yin, A. Rubio and P. M. Ajayan, Nano Lett., 2012, 12, 3518-3525.

61 X. Li, Y. Zhu, W. Cai, M. Borysiak, B. Han, D. Chen, R. D. Piner, L. Colombo and R. S. Ruoff, Nano Lett., 2009, 9, 4359-4363.

62 H. Kim, J. S. Horwitz, G. P. Kushto, Z. H. Kafafi and D. B. Chrisey, Appl. Phys. Lett., 2001, 79, 284.

63 F. L. Wong, M. K. Fung, S. W. Tong, C. S. Lee and S. T. Lee, Thin Solid Films, 2004, 466, 225-230.

64 F. Bonaccorso, Z. Sun, T. Hasan and A. C. Ferrari, Nat. Photonics, 2010, 4, 611-622.

65 J. K. Lee, C. S. Park and H. Kim, RSC Adv., 2014, 4, 6245362456.

66 M. Kim, A. Shah, C. Li, P. Mustonen, J. Susoma, F. Manoocheri, J. Riikonen and H. Lipsanen, 2D Mater., 2017, 4, 035004.

67 Y. C. Lai, B. S. Wu, S. C. Yu, P. Yu and G. C. Chi, IEEE Conf., 2013, 2436-2438.

68 C. Lee, X. Wei, J. W. Kysar and J. Hone, Science, 2008, 321, 385-388.

69 L. G. De Arco, Y. Zhang, C. W. Schlenker, K. Ryu, M. E. Thompson and C. Zhou, ACS Nano, 2010, 4, 2865-2873.

70 Z. Yin, S. Sun, T. Salim, S. Wu, X. Huang, Q. He, Y. M. Lam and H. Zhang, ACS Nano, 2010, 4, 5263-5268.

71 T. Yu, Z. Ni, C. Du, Y. You, Y. Wang and Z. Shen, J. Phys. Chem. C, 2008, 112, 12602-12605.

72 S. Takamatsu, T. Takahata, M. Muraki, E. Iwase, K. Matsumoto and I. Shimoyama, J. Micromech. Microeng., 2010, 20(1-6), 075017.

73 N. Lu, C. Lu, S. Yang and J. Rogers, Adv. Funct. Mater., 2012, 22, 4044-4050.

74 S. Lee, K. Lee, C. H. Liu and Z. Zhong, Nanoscale, 2011, 4, 639-644.

75 Quantum Wise A/S, Atomistix Tool Kit, 2010, ch. 10.8.2.

76 M. Brandbyge, J. Mozos, P. Ordejon, J. Taylor and K. Stokbro, Phys. Rev. B, 2002, 65, 165401.

77 D. M. Ceperley and B. J. Alder, Phys. Rev. Lett., 1980, 45, 566.

78 J. P. Perdew and A. Zunger, Phys. Rev. B, 1981, 23, 5048.

79 H. J. Monkhorst and J. D. Pack, Phys. Rev. B, 1976, 13, 5188. 